\title{
CDODA-Me has anticancer activity
}

CDODA-Me (methyl 2-cyano-3, 11-dioxo-18b-olean-1, 12-dien-30-oate), a triterpenoid acid found in liquorice, inhibits bladder cancer cell growth via a reactive oxygen species (ROS)-mediated mechanism, according to a study in Urologic Oncology.

CDODA-Me has previously been reported to downregulate specificity protein (Sp) transcription factors, which modulate the expression of a number of genes whose transcripts are involved in cell proliferation, survival, migration and invasion. Sp has also been shown to be dowregulated by ROS. Thus, teams from MD Anderson Cancer Center and A\&M University in Texas sought to elucidate the effects of CDODA-Me on Sp and ROS in bladder cancer cell lines.

24 hours of CDODA-Me treatment on the human bladder cancer cell lines J82, 253JB-V and RT4P was shown to reduce cell viability in a dose-dependent manner. Furthermore, CDODA-Me treatment also resulted in increased DNA fragmentation rates compared with control cells. FACS analysis demonstrated that treatment with CDODA-Me increased intracellular ROS levels,

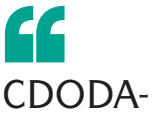
Me-induced downregulation of Sp and Sp-related genes is mediated by ROS

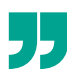
also in a dose-dependent manner, in all cell lines. As previous studies have shown that ROS can downregulate Sp and Sp-regulated proteins, the team went on to investigate the expression of Sp1, Sp3 and Sp4, showing that all three were reduced by CDODA-Me administration, and that expression of proteins known to be regulated by Sp, such as survivin, was also reduced. To determine whether the CDODA-Me-induced downregulation of Sp and Sp-related genes is mediated by ROS, the team examined DNA fragmentation following treatment with CDODA-Me alone or in combination with the antioxidant and ROS inhibitor glutathione. Coadministration inhibited the DNA fragmentation induced by CDODA-Me, suggesting that CDODA-Me's induction of apoptosis is mediated by ROS, an effect confirmed by western blotting studies.

Finally, in vivo studies were performed using $\mathrm{NCr}-\mathrm{nu} / \mathrm{nu}$ mice carrying RT4V6 xenograft tumours. CDODA-Me-treated mice had significantly smaller tumours than control mice at 39 days after injection.

Overall, this study demonstrates a compelling antineoplastic effect of CDODA-Me on bladder cancer cells, suggesting that it warrants further investigation as a potential therapy in both muscle-invasive and non-muscle-invasive bladder tumours. 